\title{
H effect on the plasticity of Ti575 alloy
}

\author{
Zhenbo Zhang, David Lunt, Philip Platt, Michael Preuss
}

School of Materials, University of Manchester, Manchester, M13 9PL, UK

\begin{abstract}
Adsorption of hydrogen $(\mathrm{H})$ can result in severe performance degradation of $\mathrm{Ti}$ alloys. In this study, the effect of $\mathrm{H}$ on the plasticity of Ti-5Al-7.5V-0.5Si (Ti575) alloy is investigated. High resolution digital image correlation (HRDIC) is employed to elucidate the effect of $\mathrm{H}$ on the strain distribution and dislocation slip character of the alloy during mechanical loading. It is found that $\mathrm{H}$ enhances slip heterogeneity and promotes the formation of long and sparse prismatic slip bands in the a phase, which can extend to across several grains. Such heterogenous prismatic slip bands are likely to originate from the lenticular hydrides, which have the prismatic plane as their habit plane. In addition, $\mathrm{H}$ also shows considerable effect on the plasticity of $\beta$ phase, which facilitates the slip transmission and enhances the slip localisation.
\end{abstract}

\section{Introduction}

$\mathrm{Ti}$ and its alloys have a high affinity to hydrogen $(\mathrm{H})$, and they can absorb large amounts of $\mathrm{H}$ when they are in contact with hydrogenating environments [1]. As with all alloys that suffer detrimental effects due to $\mathrm{H}$, Ti and its alloys also show high susceptibility to $\mathrm{H}$ embrittlement (HE). HE of Ti alloys can cause severe reduction in their toughness and unexpectedly brittle failure with considerable economical and environmental costs [1, 2]. Understanding the HE mechanism of Ti alloys is crucial for reliable lifetime prediction of components used in $\mathrm{H}$ containing environments, and performance optimisation by tailoring the microstructure and chemistry.

Several HE mechanisms for various materials have been proposed [3], and the most commonly invoked HE mechanisms for metallic materials that do not form hydrides are hydrogen-enhanced decohesion (HEDE) [4] and hydrogen-enhanced localised plasticity (HELP) [ $[\underline{]}$. However, Ti alloys are typical hydride forming systems, where cleavage fracture at hydrides and hydridesinduced interfacial decohesion also play an important role in $\mathrm{HE}[\underline{2}, \underline{6}]$. The HE of Ti alloys become further complicate by the existence of different phases, namely $\alpha$ and $\beta$ phases, and various types of phases mixtures. $\alpha$ and $\beta$ phases have very different solubility in $\mathrm{H}$ and hence very different $\mathrm{HE}$ susceptibilities $[\underline{7}, \underline{8}$. The hcp a phase has a much lower $\mathrm{H}$ solubility and more susceptible to $\mathrm{HE}$, than its allotropy bcc $\beta$ phase [1]. Therefore, in dual phase Ti alloys, hydrides usually form in the $\alpha$ phase and $\alpha / \beta$ interphase boundaries, which usually results in fracture at the interface [6]. Due to such complexities, the role of $H$ on the performance Ti alloys should be considered in different aspects: hydrides formation in the a phase and $\alpha / \beta$ interface, solute $\mathrm{H}$ in the $\alpha$ and $\beta$ phase (mainly in $\beta$ phase). Under such circumstances, the HE of Ti alloys is usually a multiple effects from different mechanisms, and current understandings on this issue is still elusive.

In this work, the $\mathrm{H}$ effect on the plasticity of a new $\alpha / \beta$ alloy TIMETAL ${ }^{\bullet} 575$ (Ti-5Al-7.5V-0.5Si, hereinafter abbreviated as Ti575) is investigated. High resolution digital image correlation (HRDIC) is employed to reveal the $\mathrm{H}$ effect on the strain development in the $\alpha$ and $\beta$ phases, in particular on the dislocation slip characters. Coupled with detailed characterisations by electron microscopy, the underlying mechanism of the $\mathrm{H}$ effect on the plasticity and $\mathrm{HE}$ of this alloy is elucidated.

\section{Experimental}

The Ti575 billet was provided by TIMET UK. The as-received material was heat treated to create a near equiaxed microstructure by annealing at $940 \mathrm{C}$ for 4 hours and then cooling at a rate of $0.75^{\circ} \mathrm{C} / \mathrm{min}$. Rectangular dog-bone tensile specimens with a gauge volume of $1.5 \times 4 \times 20 \mathrm{~mm}$ were machined using electrical discharge machining (EDM). Before $\mathrm{H}$ charging, the tensile samples were ground to P1200 grit to remove the EDM affected surface layer. Cathodic charging was carried out at $80^{\circ} \mathrm{C}$ in $0.5 \mathrm{M} \mathrm{H}_{3} \mathrm{PO}_{4}$

electrolyte under a constant current density of $50 \mathrm{~mA} / \mathrm{cm}^{2}$. The concentration of $\mathrm{H}$ in the samples was modified by altering the charging time. Eltra $9000 \mathrm{H}$ hydrogen analyser was used to obtain the bulk $\mathrm{H}$ concentration. After $\mathrm{H}$ charging, the samples were homogenised in an argon gas environment at $400^{\circ} \mathrm{C}$ for 8 hours.

High resolution strain maps were obtained from the tensile samples using high resolution digital image correlation (HRDIC) on the $\mathrm{H}$-charged sample and non-charged sample to assess the $\mathrm{H}$ effect on the strain development in the alloy. The details about HRDIC experiment and analysis can be found in previously reports [9]. Generally, HRDIC was done via three steps: 1) sample preparation; 2) imaging before and after deformation; 3) data analysis. The samples were ground and polished with OPS solution. Nanoscale gold particle patterns were put on the sample via gold sputtering and gold remodelling in water vapour at $315{ }^{\circ} \mathrm{C}$. An FEI Magellan 400 field emission gun (FEG) SEM was used for imaging before and after tensioning. Tensile tests were conducted on a Deben microtester with a $5 \mathrm{kN}$ loading cell with an initial strain rate of $1 \times 10^{-4} \mathrm{~s}^{-1}$. Cross correlation of the images prior to and after deformation was performed using La Vision DaVis software. Further data analysis on the obtained displacement data was carried out using in-house Python scripts.

Electron backscattered diffraction (EBSD) was carried out using an FEI Sirion FEG SEM to characterise the microstructures of Ti575. Transmission electron microscopy (TEM) was conducted on a FEI F30 microscope operating at $300 \mathrm{kV}$ to characterise the hydrides. TEM foils were prepared by twin-jet electropolishing (TENUPOL5, Struers), in a solution of $10 \%$ perchloric acid and $90 \%$ methanol at a temperature of $-35^{\circ} \mathrm{C}$. 


\section{Results}

Fig.1a shows the effective shear strain map obtained by HRDIC of the H-charged sample after tensioning to a strain of about $1.5 \%$. The corresponding microstructural information, including phase map, Schmid factor maps of basal slip and prismatic slip, is shown in Fig.1b-d. For comparison, a non-charged sample was tensioned to the identical strain, and similar strain map and microstructural information are shown in Fig.2. Two main differences in the slip characters can be seen in between the H-charged sample (Fig.1a) and non-charged sample (Fig.2a). In the H-charged sample, there are large amount of long slip bands that are sparsely distributed in the regions oriented with high Schmid factors for prismatic slip. These long slip bands can extend across several grain boundaries in some cases, which are not seen in the non-charged sample (Fig.2a). $\beta$ phase, which exists in between the a phase, seems to be a strong barrier for dislocation slip transmission in the non-charged sample, but such effect from $\beta$ phase is much less appreciable in the $\mathrm{H}$-charged sample. In addition, in the non-charged sample (Fig.2a), the slip bands are more diffuse and the associated strain are lower in the $\beta$ phase. These differences in the slip characters indicate a pronounced $\mathrm{H}$ effect on the plasticity of Ti575 alloy.
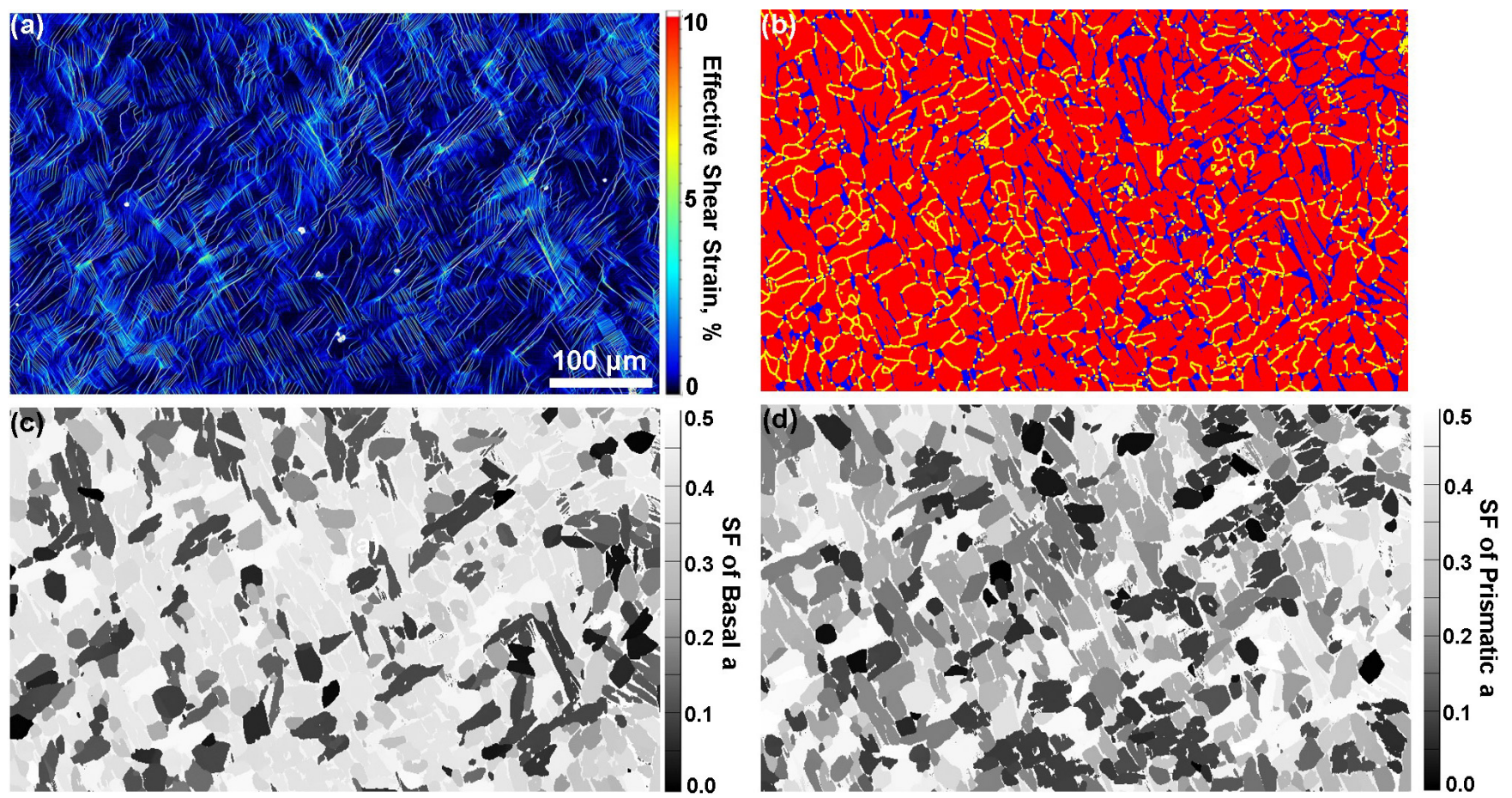

Fig.1 H-charged Ti575: (a) Effective shear strain map of the sample tensioned to about 1.5\%; (b) phase map obtained by EBSD from the same region, where red and blue regions are $\alpha$ and $\beta$ phase, respectively, and yellow lines are grain boundaries with misorientation higher than $5^{\circ}$; (c) and (d) are the corresponding Schmid Factor (SF) maps of basal and prismatic slip, respectively.
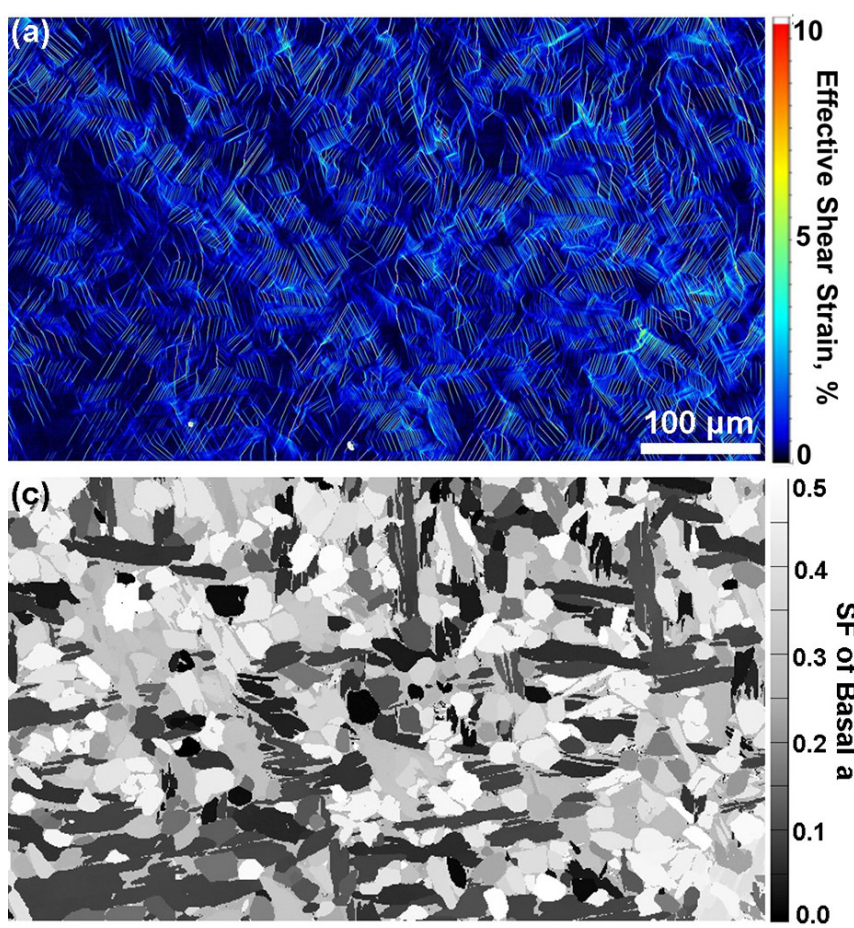
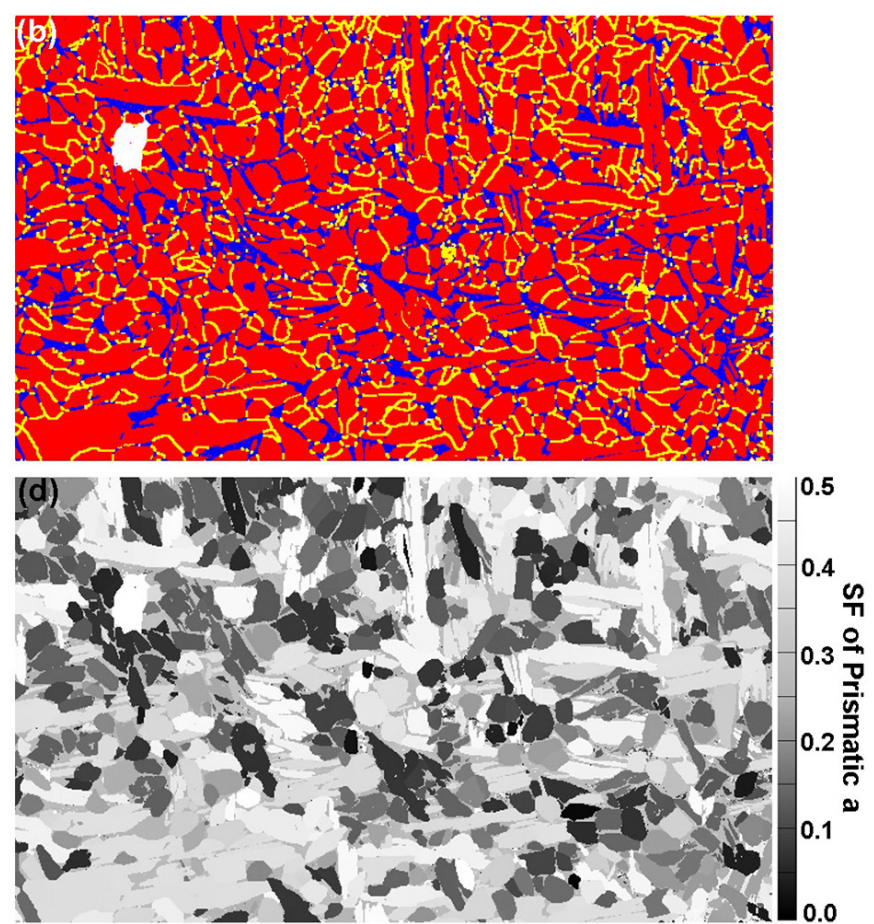
Fig.2 Non-charged Ti575: (a) Effective shear strain map of the sample tensioned to about 1.5\%; (b) phase map obtained by EBSD from the same region, where red and blue regions are $\alpha$ and $\beta$ phase, respectively, and yellow lines are grain boundaries with misorientation higher than $5^{\circ} ;$ (c) and (d) are the corresponding Schmid Factor (SF) maps of basal and prismatic slip, respectively.

Fig.3a and $3 \mathrm{~b}$ show the shear strain maps of the H-charged and non-charged samples at a higher magnification. The differences on the above-mentioned aspects between them are clearly visualised. Analysis on the slip character of the long and sparse slip bands in the H-charged samples indicate that the majority of them are prismatic slip bands. Two examples of them are shown in Fig.3c and 3d. Both slip traces are identified as prismatic slip without any ambiguity, and the Schmid factors of the predicted slip systems are 0.47 and 0.39 , respectively.
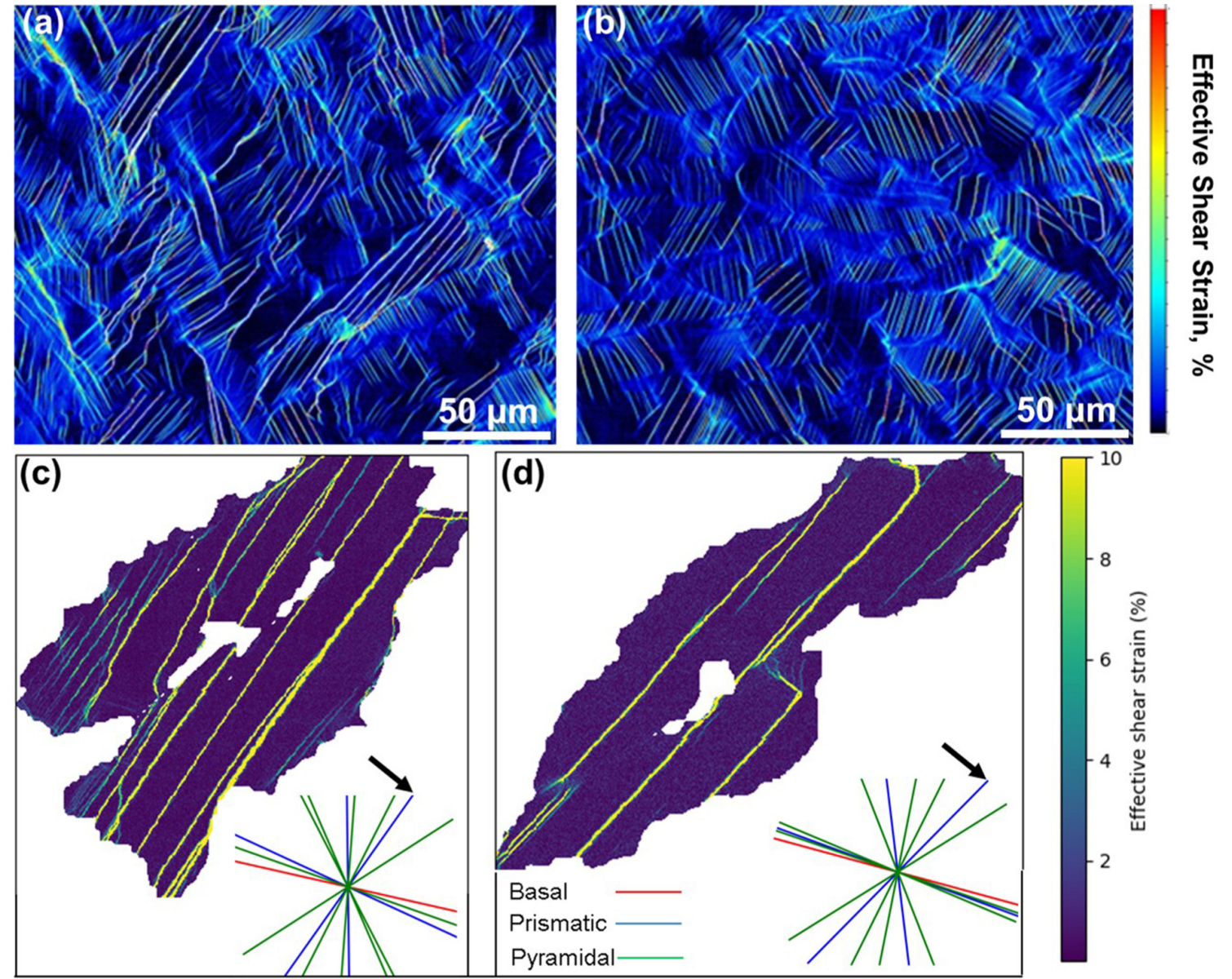

Fig.3 (a) and (b) Effective shear strain maps of the H-charged and non-charged samples, respectively; (c) and (d) two examples showing the slip system analysis of grains with long and sparse slip bands in the H-charged sample, and the arrows indicate the active slip system.

The $\mathrm{H}$ effect on the slip characters of Ti575 alloy is also reflected in the effective shear strain distribution plot, as shown in Fig.4. The difference in the frequency distribution between the H-charged and non-charged sample is evident. Specifically, the frequency distribution for the non-charged sample is narrower and the frequency near the mean effective shear strain is higher, which indicates a more homogeneous strain distribution. In both the low and high shear strains ranges, the frequency is higher for the $\mathrm{H}$-charged sample. This is very likely due to the higher frequency of long slip bands with high shear strain in the $\mathrm{H}$-charged sample, which is consistent with the differences in the slip characters seen in the shear strain maps. 


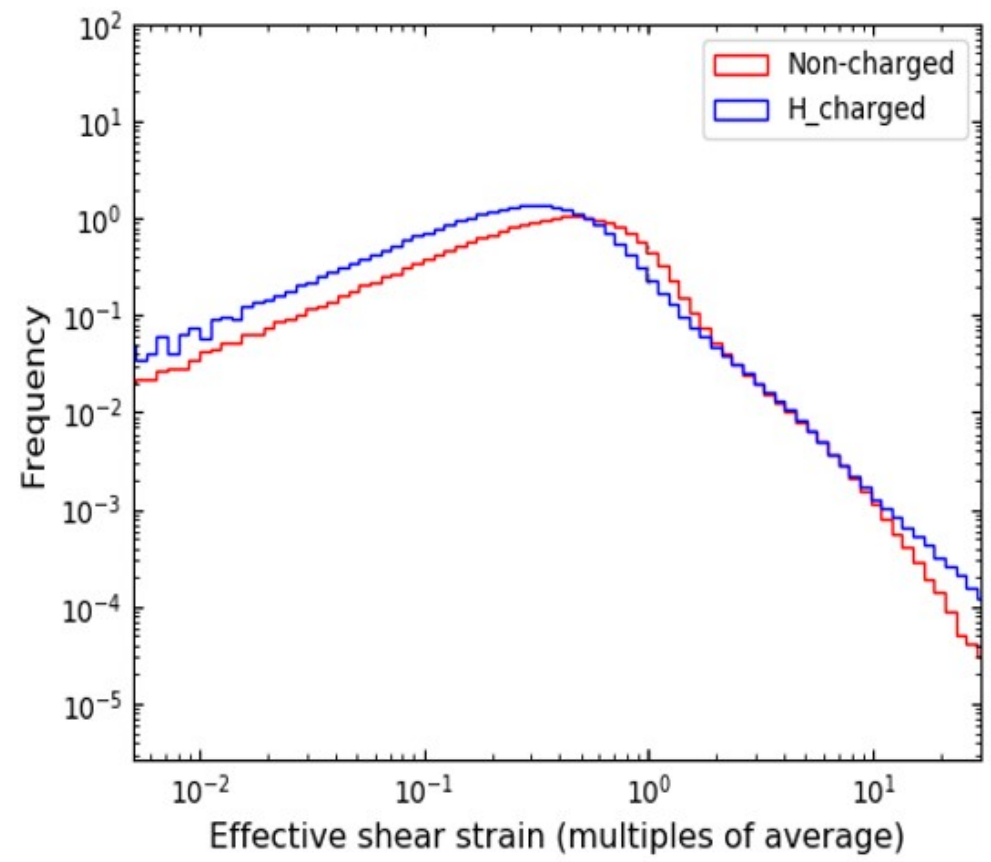

Fig.4 Normalised frequency distribution of the normalised effective shear strain of the H-charged and non-charged samples obtained by HRDIC

The mechanical response of Ti575 alloy is also affected by the presence of H. Fig. 5 shows the work hardening behaviours of the samples with different $\mathrm{H}$ contents. It is seen that the work hardening rates of the $\mathrm{H}$ charged samples are considerably lower than that of non-charged samples at the early stage of work hardening. This is consistent with the above HRDIC results, since higher strain heterogeneity and more pronounced strain localisation can lead to a lower work hardening rate of the H-charged sample, which is discussed later.

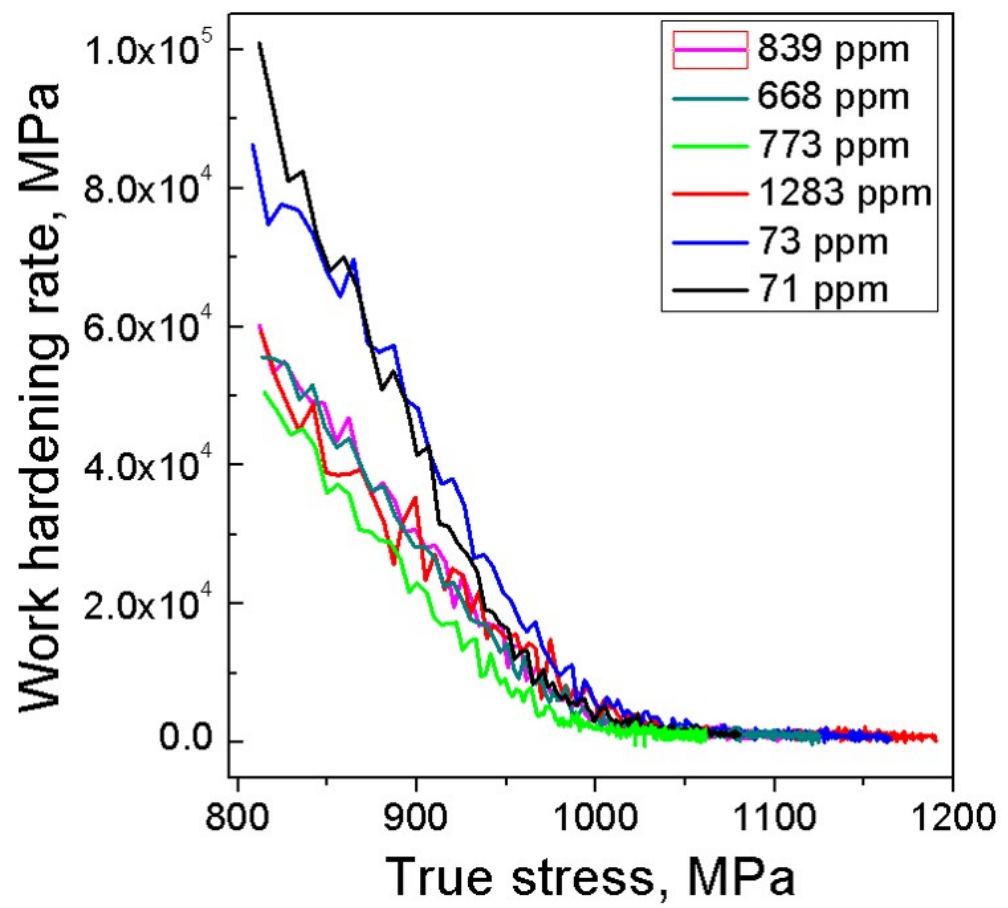

Fig. 5 Work hardening rate versus true stress plots of the Ti575 samples with different $\mathrm{H}$ content. Note that the samples with $71 \mathrm{ppm}$ and 73 ppm $\mathrm{H}$ are non-charged.

\section{Discussion}

$\mathrm{H}$ shows considerable effects on the plasticity of Ti575 alloy in the form of enhanced slip heterogeneity and strain localisation, which mainly originates from the $\mathrm{H}$ induced heterogeneous prismatic slip of the a phase. Since a phase has very low $\mathrm{H}$ solubility, the majority of $\mathrm{H}$ in the a phase is supposed to form hydrides or diffuses into $\beta$ phase during homogenisation treatment. Therefore, hydrides should play a dominant role in influencing the plasticity of a phase. 
TEM was performed to characterise the hydrides, and an example is shown in Fig.6. The hydride is identified as $\delta$ hydride with face centred cubic structure. The orientation relationship between the hydride and the a matrix is $[0001]_{\alpha} \|[001]_{\mathrm{TiH}}$ and $(1-$ 100) $\mathrm{a}_{\|}(2-20)_{\mathrm{TiH},}$ which is consistent to the orientation relationship reported in the literature [10]. It can also be seen that the habit plane of the lenticular hydride is along the $\{1-100\}$ plane, i.e. the prismatic plane. Dislocations have the tendency to activate and slip along the hydrides habit plane, as invoked in some previous studies $[11, \underline{12}]$. This correlation is suggested to be a consequence of a significant volume expansion $(20 \%)$ on forming hydride from the $\alpha$ phase, which is largely accommodated by plastic deformation at the interfacial regions $[\underline{6}, \underline{11}, \underline{12}]$. This is suggested to be the main reason for the more heterogeneous long prismatic slip bands developed in the H-charged sample.
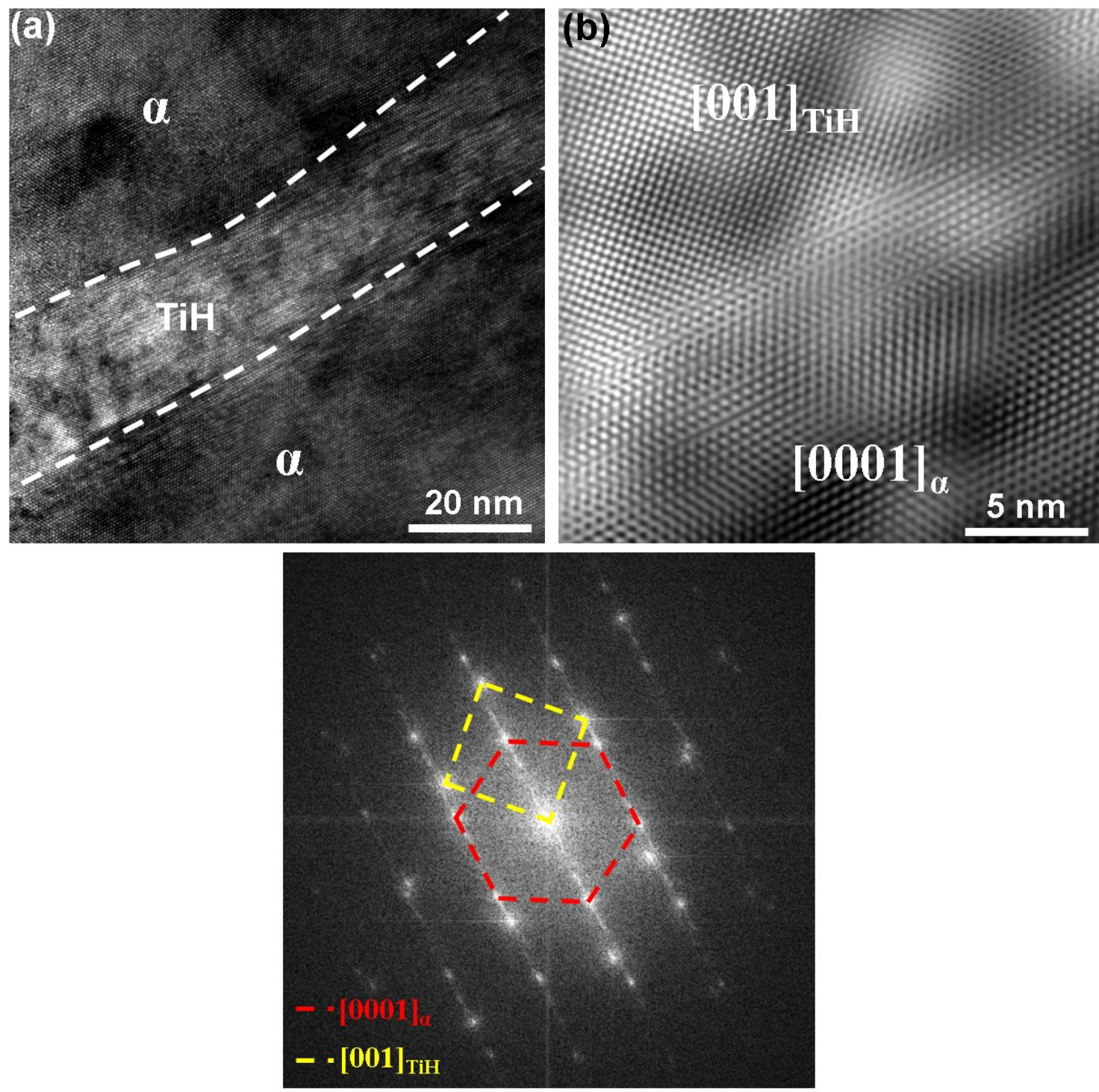

Fig.6 TEM analysis of the hydride in Ti575: (a) HRTEM image showing $\delta$-TiH and a matrix; (b) IFFT image from a region in (a) showing the interface between $\delta$-TiH and a matrix; (c) FFT pattern illustrating the orientation relationship between $\delta$-TiH and $a$ matrix.

The plastic deformation of $\beta$ phase seems to be also affected by the presence of $\mathrm{H}$. As shown in Fig.3, $\beta$ phase acts as strong barrier for slip transmission in the non-charged sample. However, such phenomenon is much less appreciable in the $\mathrm{H}$-charged sample. $\beta$ phase can store a high content of $\mathrm{H}$ in solution due to its high solubility in $\mathrm{H}$. Substantial $\mathrm{H}$ is reported to have a softening effect on $\beta$ phase, which can be explained under the framework of HELP mechanism [ $\underline{3}, \underline{7}]$. Under such circumstances, $\beta$ phase becomes weaker in terms of preventing slip transmission among a grains. $H$ can even enhance the localised plasticity of $\beta$ grains [?], which can potentially promotes the slip transmission and hence results in the long slip bands across several grains. As a result, strain hardening is reduced as observed in Fig. 5.

\section{Conclusion}

Hydrogen shows considerable effect on the plasticity of the Ti575 alloy. Strain analysis using HRDIC technique demonstrates that $\mathrm{H}$ largely enhances slip heterogeneity and promotes the formation of long and sparse prismatic slip bands in the a phase. It seems that the heterogenous slip behaviours of the $\mathrm{H}$-charged sample originates from the lenticular hydrides, which have the prismatic plane as their habit plane. Moreover, the mechanism of hydrogen enhanced localised plasticity seems to work for $\beta$ phase, which facilitates the slip transmission among a grains and enhances the slip localisation, therefore reducing strain hardening in the material. 


\section{Acknowledgements}

The authors would like to thank the HEXMAT (Heterogeneous Mechanics in Hexagonal Alloys across Length and Time Scales) programme grant [EP/K034332/1]. Michael Preuss is grateful for the EPSRC Leadership Fellowship grant [EP/I005420/1]. The authors acknowledge the TIMET UK for providing the alloy.

\section{$\underline{\text { References }}$}

[1] C.L. Briant, Z.F. Wang, N. Chollocoop, Hydrogen embrittlement of commercial purity titanium, Corros. Sci. 44 (2002) $1875-1888$.

[2] T.P. Chapman, D. Dye, D. Rugg, Hydrogen in Ti and Zr alloys: industrial perspective, failure modes and mechanistic understanding, Philosophical Transactions of the Royal Society A: Mathematical, Physical and Engineering Sciences 375 (2017) 20160418.

[3] I.M. Robertson, P. Sofronis, A. Nagao, M.L. Martin, S. Wang, D.W. Gross, K.E. Nygren, Hydrogen Embrittlement Understood, Metall. Mater. Trans. A 46A (2015) 2323-2341.

[4] D. Tromans, On Surface-Energy and the Hydrogen Embrittlement of Iron and Steels, Acta Metall. Mater. 42 (1994) $2043-$ 2049.

[5] P.J. Ferreira, I.M. Robertson, H.K. Birnbaum, Hydrogen effects on the interaction between dislocations, Acta Mater. 46 (1998) 1749-1757.

[6] D.S. Shih, I.M. Robertson, H.K. Birnbaum, Hydrogen embrittlement of a titanium: In situ tem studies, Acta Metall. 36 (1988) 111-124.

[7] A.M. Alvarez, I.M. Robertson, H.K. Birnbaum, Hydrogen embrittlement of a metastable $\beta$-titanium alloy, Acta Mater. 52 (2004) 4161-4175.

[8] D.F. Teter, I.M. Robertson, H.K. Birnbaum, The effects of hydrogen on the deformation and fracture of $\beta$-titanium, Acta Mater. 49 (2001) 4313-4323.

[9] F. Di Gioacchino, J. Quinta da Fonseca, An experimental study of the polycrystalline plasticity of austenitic stainless steel, Inter. J. Plasticity 74 (2015) 92-109.

[10] E. Conforto, I. Guillot, X. Feaugas, Solute hydrogen and hydride phase implications on the plasticity of zirconium and titanium alloys: a review and some recent advances, Philosophical Transactions of the Royal Society A: Mathematical, Physical and Engineering Sciences 375 (2017) 20160417.

[11] H. Numakura, M. Koiwa, Hydride precipitation in titanium, Acta Metall. 32 (1984) 1799-1807.

[12] N.E. Paton, B.S. Hickman, D.H. Leslie, Behavior of hydrogen in a-phase Ti-Al alloys, Metall. Trans. 2 (1971) $2791-2796$. 